\title{
A Comparative Study between Bedside Chest Ultrasound and Traditional Chest Radiography in Critically Ill Patients
}

\author{
Omar M. Abo Shahba, Mohamed F. Agag, Badr I. Allah, Shadi M. Yousef
}

\author{
Department of Anesthesia and ICU, Faculty of Medicine, Al-Azhar University, Cairo, Egypt \\ Corresponding author: Shadi M. Yousef; Mobile: 01095266156; Email: shadi_magdi_1990@hotmail.com
}

\begin{abstract}
Background: Bedside chest ultrasound emerge a sensitive tool with much higher sensitivity than plain chest $\mathrm{x}$ ray. It avoids the cost, risk of transportation to perform the CT chest and that of radiation exposure. Aim of the Study: was to evaluate the sensitivity and the specificity of bedside ultrasound in the detection and the evaluation of chest conditions of critically ill patients in comparison of CT chest as the gold standard tool. Patients and Methods: This prospective study was conducted on a registry of 80 patients with different chest pathology admitted to the Medical-Surgical Intensive Care Unit of Bab Al Shaeria Hospital between March and November 2018. Results: bedside chest ultrasound sensitivity in detection and evaluation of pneumothorax, pleural effusion, and consolidation and Interstitial lung disease in comparison to CT chest showed sensitivity around $93 \%$ with little cost, without risk of radiation exposure and transportation of critically ill patients. Conclusion: Bedside chest ultrasound is a highly sensitive and specific tool in the detection and evaluation of many chest conditions that eliminates the risk of transportation of unstable patients and that of radiation exposure and is easily accessible in resource limited areas.
\end{abstract}

Key words: bedside chest ultrasound, traditional chest radiography, critically ill patients

\section{Introduction:}

Traditionally chest imaging in critically ill patients is performed either by bedside chest radiography (CXR) or thoracic computed tomography (CT), both techniques have limitations which constrain their usefulness ${ }^{(1)}$.

Although thoracic CT is the gold standard for chest imaging, it is expensive and cannot be performed on a routine basis. In addition to the transportation of critically ill patients to the radiology department combined with the radiation exposure carries a measurable risk ${ }^{(2)}$.

On the other hand, limitations of bedside CXR have been well described and lead to poorquality X-ray films with low sensitivity. Indeed, it has been shown that even under carefully controlled exposure conditions more than $30 \%$ of the X-ray films are considered suboptimal ${ }^{(3)}$.

Finally, there is poor correlation between CXR findings and those of CT. Nevertheless, despite these limitations bedside CXR remains the daily reference for chest imaging, Nowadays bedside chest ultrasound is increasingly used in patients managed in intensive care units (ICUs) ${ }^{(4)}$.

It has been shown in patients with acute respiratory distress syndrome (ARDS) that compared to bedside chest X-ray, chest ultrasound has a higher diagnostic accuracy for pleural effusion, consolidation, and interstitial syndrome (5).

The aim of the current study was to compare the diagnostic performance of chest ultrasound versus bedside chest CXR and thoracic C.T for the detection of several pathologic abnormalities in unselected critically ill patients.

\section{Patients and Methods:}

This prospective study included a total of 80 patients with different chest pathology admitted to the Medical-Surgical Intensive Care Unit of Bab AlShaeria Hospital. Approval of the ethical committee and a written informed consent from all the subjects were obtained. This study was conducted between March and November 2018. Patients were assigned into four groups. The groups are categorized after the results of radiological investigations and allocated randomly as the following:
a) Group A, 20 patients with pneumothorax.
b) Group B, 20 patients with pleural effusion.
c) Group C, 20 patients with consolidation.
d) Group D, 20 patients with interstitial chest syndrome.
Patients age was ranging from 22-85 years, $74 \%$ of them were males. $46 \%$ of patients were diabetic while $42 \%$ were chronic hypertensive. The demographic data for the included patients is demonstrated in table (1).

\section{Inclusion criteria:}

This study included any patient with different chest pathology regardless the age of adult ICU patient or gender. Chest lesions included consolidation, pnemothorax, pleural effusion and interstitial chest syndrome. 


\section{Exclusion criteria:}

- Patients who don't undergo CT-Chest for not being stable for transportation.

- Patients who have a life threating problem (Tension pneumothorax).

- Patients with limit imaging acquisition are related to surgical injury and chest dressings that can alter or preclude the transmission of ultrasound beams to the lung.

- Other pathology rather than four pathology we mentioned.

Patients were enrolled in the study when a thoracic CT scan with iodine contrast material was ordered by the primary physician not involved in the study. No other selection criteria were applied.

Prior to CT scan a bedside CXR was obtained and lung ultrasound was performed. Ventilator settings were kept unchanged throughout the study in patients who are mechanically ventilated.

Four pathologic entities were evaluated by each imaging method: consolidation, interstitial syndrome, pneumothorax, and pleural effusion.

For data analysis each hemithorax was divided into six regions (see below), three in upper fields (anterior, posterior, lateral) and three in lower fields (anterior, posterior, lateral).

\section{Chest radiography}

Anterior CXR was performed using portable X-ray equipment (SMAM X-ray equipment - roller 30). The evaluation of CXR was performed by an expert radiologist, unaware of the lung ultrasound and CT findings.

\section{Lung ultrasound}

Visualization of the lungs was performed using a microconvex 5-9 $\mathrm{MHz}$ transducer appropriate for transthoracic examination (Toshiba Xario 200).

Access to standardized images (seashore sign, stratosphere sign) was possible.

Ultrasonography was randomly and blindly evaluated by a single operator, who was unaware of the CT and CXR findings.

Lungs were divided into 12 regions as previously described ${ }^{(6)}$.

\section{Statistical analysis:}

Recorded data were analyzed using the statistical package for social sciences, version 20.0 (SPSS Inc., Chicago, Illinois, USA). Quantitative data were expressed as mean \pm standard deviation (SD). Qualitative data were expressed as frequency and percentage.

\section{The following tests were done:}

- Chi-square $\left(\mathrm{x}^{2}\right)$ test of significance was used in order to compare proportions between qualitative parameters.

- Evaluation of Diagnostic Performance:

- Sensitivity $=($ true $+v e) /[($ true $+v e)+$ (false-ve)].

- $\quad$ Specificity $=($ true $-v e) /[($ true $-v e)+$ (false +ve)].

- $\quad P P V=($ true $+v e) /[($ true $+v e)+($ false $+\mathrm{ve})]$.

- $\quad \mathrm{NPV}=($ true $-\mathrm{ve}) /[($ true $-\mathrm{ve})+($ false ve)].

- $\quad$ Accuracy $=(\mathrm{TP}+\mathrm{TN}) /[\mathrm{TP}+\mathrm{FP}+\mathrm{TN}+\mathrm{FN}]$

- The confidence interval was set to $95 \%$ and the margin of error accepted was set to $5 \%$. So, the $\mathrm{p}$-value was considered significant as the following:

- Probability (P-value)

- P-value $<0.05$ was considered significant.

- P-value <0.001 was considered as highly significant.

- P-value >0.05 was considered insignificant.

\section{Results}

The results of the present study are demonstrated in the following tables:

Table (1): Demographic data distribution of the study group.

\begin{tabular}{|l|l|}
\hline Demographic data & Total $(\mathbf{n}=\mathbf{8 0})$ \\
\hline Sex & \\
Female & $21(26.25 \%)$ \\
Male & $59(73.75 \%)$ \\
\hline Age (years) & $22-85$ \\
Range & $55.06 \pm 13.61$ \\
Mean \pm SD & \\
\hline Other Comorbidity & $37(46.25 \%)$ \\
DM & $34(42.50 \%)$ \\
HTN & \\
\hline Pathology & $20(25.00 \%)$ \\
Pnemothorax & $20(25.00 \%)$ \\
Pleural effusion & $20(25.00 \%)$ \\
Consolidation & $20(25.00 \%)$ \\
\hline Interstitial lung disease & \\
\hline
\end{tabular}


This table shows that the Female were $21(26.25 \%)$ while Male were $59(73.75 \%)$. The patients age (years) Ranged 22-85 and Mean \pm SD 55.06 \pm 13.61 , Other Comorbidity DM 37 (46.25\%), HTN 34 (42.50\%), Pathology Pnemothorax 20 (25.00\%), Pleural effusion 20 (25.00\%), Consolidation 20 (25.00\%) and Interstitial lung disease $20(25.00 \%)$

Table (2): Pnemothorax distribution of the study group.

\begin{tabular}{|l|l|l|}
\hline Pnemothorax & No. & $\%$ \\
\hline X-ray & & \\
Positive & 9 & $45.0 \%$ \\
Negative & 11 & $55.0 \%$ \\
\hline Ultrasound & & \\
Positive & 16 & $80.0 \%$ \\
Negative & 4 & $20.0 \%$ \\
\hline CT & 16 & $80.0 \%$ \\
Positive & 4 & $20.0 \%$ \\
Negative & 4 & \\
\hline
\end{tabular}

This table shows that the x-ray Positive (45.0\%), Negative (55.0\%), Ultrasound Positive (80.0\%), Negative (20.0\%), CT Positive (80.0\%) and Negative (20.0\%) of pneumothorax.

Table (3): Pleural effusion distribution of the study group.

\begin{tabular}{|l|l|l|}
\hline Pleural effusion & No. & $\%$ \\
\hline x-ray & & \\
Positive & 12 & $60.0 \%$ \\
Negative & 8 & $40.0 \%$ \\
\hline Ultrasound & & \\
Positive & 16 & $80.0 \%$ \\
Negative & 4 & $20.0 \%$ \\
\hline CT & & \\
Positive & 16 & $80.0 \%$ \\
Negative & 4 & $20.0 \%$ \\
\hline
\end{tabular}

This table shows that the x-ray Positive (60.0\%), Negative (40.0\%), Ultrasound Positive (80.0\%), Negative (20.0\%), CT Positive (80.0\%) and Negative (20.0\%) of pleural effusion.

Table (4): Consolidation distribution of the study group.

\begin{tabular}{|l|l|l|}
\hline Consolidation & No. & $\%$ \\
\hline x-ray & & \\
Positive & 12 & $60.0 \%$ \\
Negative & 8 & $40.0 \%$ \\
\hline Ultrasound & & \\
Positive & 16 & $80.0 \%$ \\
Negative & 4 & $20.0 \%$ \\
\hline CT & & \\
Positive & 15 & $75.0 \%$ \\
Negative & 5 & $25.0 \%$ \\
\hline
\end{tabular}

This table shows that the x-ray Positive $(60.0 \%)$, Negative $(40.0 \%)$, Ultrasound Positive $(80.0 \%)$, Negative $(20.0 \%)$, CT Positive (75.0\%) and Negative (25.0\%) of consolidation.

Table (5): Interstitial lung disease distribution of the study group.

\begin{tabular}{|l|l|l|}
\hline Interstitial lung disease & No. & $\%$ \\
\hline x-ray & & \\
Positive & 7 & $35.0 \%$ \\
Negative & 13 & $65.0 \%$ \\
\hline Ultrasound & & \\
Positive & 15 & $75.0 \%$ \\
Negative & 5 & $25.0 \%$ \\
\hline CT & 15 & $75.0 \%$ \\
Positive & 5 & $25.0 \%$ \\
Negative & 5 & P. \\
\hline
\end{tabular}

This table shows that the x-ray Positive (35.0\%), Negative (65.0\%), Ultrasound Positive (75.0\%), Negative (25.0\%), CT Positive (75.0\%) and Negative (25.0\%) of interstitial lung disease. 
Table (6): Pathology distribution of the study group.

\begin{tabular}{|l|l|}
\hline Total Pathology & Total $(\mathbf{n}=\mathbf{8 0})$ \\
\hline Chest x-ray & \\
Positive & $40(50.00 \%)$ \\
Negative & $40(50.00 \%)$ \\
\hline Chest U/S & \\
Positive & $63(78.80 \%)$ \\
Negative & $17(21.30 \%)$ \\
\hline Thoracic CT & \\
Positive & $62(77.50 \%)$ \\
Negative & $18(22.50 \%)$ \\
\hline
\end{tabular}

This table shows that the x-ray Positive (50.0\%), Negative (50.0\%), Ultrasound Positive (78.8\%), Negative (21.30\%), CT Positive (77.50\%) and Negative (22.5\%) of pathology.

Table (7): Sensitivity, specificity, positive and negative predictive values, and diagnostic accuracy of x-ray and ultrasound compared to CT scan for pneumothorax.

\begin{tabular}{|c|c|c|c|c|c|c|c|}
\hline \multirow{3}{*}{ Pneumothorax } & \multicolumn{2}{|c|}{ CT } & \multirow{3}{*}{ Sens. } & \multirow{3}{*}{ Spec. } & \multirow{3}{*}{ PPV } & \multirow{3}{*}{ NPV } & \multirow{3}{*}{ Accuracy } \\
\hline & Positive & Negative & & & & & \\
\hline & $N=16$ & $\mathrm{~N}=4$ & & & & & \\
\hline \multicolumn{8}{|l|}{ x-ray } \\
\hline Positive & $\mathrm{TP}=7$ & $\mathrm{FP}=2$ & \multirow{3}{*}{$43.8 \%$} & \multirow{2}{*}{$50.0 \%$} & \multirow{2}{*}{$77.8 \%$} & \multirow{2}{*}{$18.2 \%$} & \multirow{2}{*}{$45.0 \%$} \\
\hline Negative & $\mathrm{FN}=9$ & $\mathrm{TN}=2$ & & & & & \\
\hline \multicolumn{7}{|l|}{ Ultrasound } & \\
\hline Positive & $\mathrm{TP}=15$ & $\mathrm{FP}=1$ & \multirow{2}{*}{$93.8 \%$} & \multirow{2}{*}{$75.0 \%$} & \multirow{2}{*}{$93.8 \%$} & \multirow{2}{*}{$75.0 \%$} & \multirow{2}{*}{$90.0 \%$} \\
\hline Negative & $\mathrm{FN}=1$ & $\mathrm{TN}=3$ & & & & & \\
\hline
\end{tabular}

- Diagnostic accuracy of x-ray and compared to CT scan for pneumothorax with sensitivity of $43.8 \%$ specificity of $50 \%$ positive predictive value of $77.8 \%$, negative predictive value of $18.2 \%$ with diagnostic accuracy of $45 \%$, with p-value (0.736 non-significant).

- Diagnostic accuracy of ultrasound and compared to CT scan for pneumothorax with sensitivity of $93.8 \%$ specificity of $75 \%$ positive predictive value of $93.8 \%$, negative predictive value of $75 \%$ with diagnostic accuracy of $90 \%$, with p-value (0.018 significant).

Table (8): Sensitivity, specificity, positive and negative predictive values, and diagnostic accuracy of CXR and ultrasound compared to CT scan for pleural effusion.

\begin{tabular}{|c|c|c|c|c|c|c|c|}
\hline \multirow{3}{*}{ Pleural effusion } & \multicolumn{2}{|c|}{ CT } & \multirow{3}{*}{ Sens. } & \multirow{3}{*}{ Spec. } & \multirow{3}{*}{ PPV } & \multirow{3}{*}{ NPV } & \multirow{3}{*}{ Accuracy } \\
\hline & Positive & Negative & & & & & \\
\hline & $N=16$ & $N=4$ & & & & & \\
\hline \multicolumn{8}{|l|}{ x-ray } \\
\hline Positive & $\mathrm{TP}=10$ & $\mathrm{FP}=2$ & \multirow{2}{*}{$62.5 \%$} & \multirow{2}{*}{$50.0 \%$} & \multirow{2}{*}{$83.3 \%$} & \multirow{2}{*}{$25.0 \%$} & \multirow{2}{*}{$60.0 \%$} \\
\hline Negative & $\mathrm{FN}=6$ & $\mathrm{TN}=2$ & & & & & \\
\hline \multicolumn{8}{|l|}{ Ultrasound } \\
\hline Positive & $\mathrm{TP}=15$ & $\mathrm{FP}=1$ & \multirow{2}{*}{$93.8 \%$} & \multirow{2}{*}{$75.0 \%$} & \multirow{2}{*}{$93.8 \%$} & \multirow{2}{*}{$75.0 \%$} & \multirow{2}{*}{$90.0 \%$} \\
\hline Negative & $\mathrm{FN}=1$ & $\mathrm{TN}=3$ & & & & & \\
\hline
\end{tabular}

- Diagnostic accuracy of x-ray and compared to CT scan for pleural effusion with sensitivity of $62.5 \%$ specificity of $50 \%$ positive predictive value of $83.3 \%$, negative predictive value of $25 \%$ with diagnostic accuracy of $60 \%$, with p-value (0.909 non-significant).

- Diagnostic accuracy of ultrasound and compared to CT scan for pleural effusion with sensitivity of $93.8 \%$ specificity of $75 \%$ positive predictive value of $93.8 \%$, negative predictive value of $75 \%$ with diagnostic accuracy of $90 \%$, with p-value (0.018 significant).

Table (9): Sensitivity, specificity, positive and negative predictive values, and diagnostic accuracy of CXR and ultrasound compared to CT scan for consolidation.

\begin{tabular}{|c|c|c|c|c|c|c|c|}
\hline \multirow[b]{2}{*}{ Consolidation } & \multicolumn{2}{|c|}{ CT } & \multirow[b]{2}{*}{ Sens. } & \multirow[b]{2}{*}{ Spec. } & \multirow[b]{2}{*}{ PPV } & \multirow[b]{2}{*}{ NPV } & \multirow[b]{2}{*}{ Accuracy } \\
\hline & $\begin{array}{c}\text { Positive } \\
\mathrm{N}=15\end{array}$ & $\begin{array}{c}\text { Negative } \\
\mathrm{N}=5\end{array}$ & & & & & \\
\hline \multicolumn{8}{|l|}{$\mathrm{x}$-ray } \\
\hline Positive & $\mathrm{TP}=8$ & $\mathrm{FP}=4$ & \multirow{3}{*}{$53.3 \%$} & \multirow{2}{*}{$20.0 \%$} & \multirow{2}{*}{$66.7 \%$} & \multirow{2}{*}{$12.5 \%$} & \multirow{2}{*}{$45.0 \%$} \\
\hline Negative & $\mathrm{FN}=7$ & $\mathrm{TN}=1$ & & & & & \\
\hline \multicolumn{7}{|l|}{ Ultrasound } & \\
\hline Positive & $\mathrm{TP}=14$ & $\mathrm{FP}=2$ & \multirow{2}{*}{$93.3 \%$} & \multirow{2}{*}{$60.0 \%$} & \multirow{2}{*}{$87.5 \%$} & \multirow{2}{*}{$75.0 \%$} & \multirow{2}{*}{$85.0 \%$} \\
\hline Negative & $\mathrm{FN}=1$ & $\mathrm{TN}=3$ & & & & & \\
\hline
\end{tabular}


- Diagnostic accuracy of x-ray and compared to CT scan for consolidation with sensitivity of 53.3\% specificity of $20 \%$ positive predictive value of $66.7 \%$, negative predictive value of $12.5 \%$ with diagnostic accuracy of $45 \%$, with p-value ( 0.278 non-significant).

- Diagnostic accuracy of ultrasound and compared to CT scan for consolidation with sensitivity of $93.3 \%$ specificity of $60 \%$ positive predictive value of $87.5 \%$, negative predictive value of $75 \%$ with diagnostic accuracy of $85 \%$, with p-value (0.048 significant).

Table (10): Sensitivity, specificity, positive and negative predictive values, and diagnostic accuracy of CXR and ultrasound compared to CT scan for interstitial lung disease.

\begin{tabular}{|c|c|c|c|c|c|c|c|}
\hline \multirow{3}{*}{ Interstitial lung disease } & \multicolumn{2}{|c|}{ CT } & \multirow{3}{*}{ Sens. } & \multirow{3}{*}{ Spec. } & \multirow{3}{*}{ PPV } & \multirow{3}{*}{ NPV } & \multirow{3}{*}{ Accuracy } \\
\hline & Positive & Negative & & & & & \\
\hline & $\mathrm{N}=15$ & $\mathrm{~N}=5$ & & & & & \\
\hline \multicolumn{8}{|l|}{ x-ray } \\
\hline Positive & $\mathrm{TP}=4$ & $\mathrm{FP}=3$ & \multirow{2}{*}{$26.7 \%$} & \multirow{2}{*}{$40.0 \%$} & \multirow{2}{*}{$57.1 \%$} & \multirow{2}{*}{$15.4 \%$} & \multirow{2}{*}{$30.0 \%$} \\
\hline Negative & $\mathrm{FN}=11$ & $\mathrm{TN}=2$ & & & & & \\
\hline \multicolumn{8}{|l|}{ Ultrasound } \\
\hline Positive & $\mathrm{TP}=14$ & $\mathrm{FP}=1$ & \multirow{2}{*}{$93.3 \%$} & \multirow{2}{*}{$80.0 \%$} & \multirow{2}{*}{$93.3 \%$} & \multirow{2}{*}{$80.0 \%$} & \multirow{2}{*}{$90.0 \%$} \\
\hline Negative & $\mathrm{FN}=1$ & $\mathrm{TN}=4$ & & & & & \\
\hline
\end{tabular}

- Diagnostic accuracy of $\mathbf{x}$-ray and compared to CT scan for interstitial lung disease with sensitivity of $26.7 \%$ specificity of $40 \%$ positive predictive value of $57.1 \%$, negative predictive value of $12.4 \%$ with diagnostic accuracy of $30 \%$, with p-value (0.659 non-significant).

- Diagnostic accuracy of ultrasound and compared to CT scan for interstitial lung disease with sensitivity of $93.3 \%$ specificity of $80 \%$ positive predictive value of $93.3 \%$, negative predictive value of $80 \%$ with diagnostic accuracy of $90 \%$, with p-value (0.007 significant).

\section{Discussion}

The main finding of this study is that in a mixed surgical- medical ICU critically ill patients, lung ultrasound identifies the most common pathologic abnormalities of the respiratory system encountered in these patients with high diagnostic accuracy.

It is important to note that patients were enrolled in the study when a thoracic CT scan was ordered by the primary physician and no other selection criteria were applied. Therefore, the lung ultrasound performance was tested in unselected critically ill patients with a variety of conditions that might interfere with the imaging technique (obesity, chest trauma, tissue edema).

This study evaluated four common abnormalities (pneumothorax, pleural effusions, consolidation, and interstitial syndrome) that have important implications in patient management and decision making. In order to accurately characterize the diagnostic performance of the three imaging methods 12 lung regions were analyzed in each patient. Nevertheless, in the decision-making process the presence of certain pathology in a hemithorax is more important than its exact localization.
In addition, posture may affect the location of the findings making the clinical significance of analysis of relatively small lung regions questionable. For this reason, we compared findings of all imaging modalities in each hemithorax.

This study proved that this analysis is clinically more relevant than the analysis of each separate region and at the same time minimizes the effects of posture (i.e., gravity) and the problem of overlapping between adjunct regions on the diagnostic performance of the various imaging techniques.

\section{A) Pneumothorax:}

The bedside diagnosis of pneumothorax is extremely important in ICU patients. It is known that supine CXR is not sensitive for diagnosis of pneumothorax, in addition to the low radiogencity of the portable x-ray.

Indeed in this study bedside CXR identified only 9 patients with pneumothorax from 20 patients with number of false positive and negative results. This may be a result from bad quality or bad positioning of patients.

Lung ultrasound has been successfully used for identification of pneumothorax in a variety of patients. These studies report a sensitivity of $93.8 \%$.

In this study lung ultrasound identified sixteen out of the twenty pneumothoraxes, one false positive result occurred in patient with subcutaneous emphysema due to chest trauma.

The one false negative result of pneumothorax missed by lung ultrasound was small and none required drainage nevertheless, because lung ultrasound did not miss any clinically significant pneumothorax, our data indicate that this technique is a reliable tool for bedside diagnosis of this abnormality. 


\section{Pleural effusion:}

In this study bedside CXR identified only 12 patients with pleural effusion from 20 patients with number of false positive and negative results. This may result from bad quality or bad positioning of patients, Accuracy of CXR was $45 \%$ indicating that this technique is not sufficient in diagnosis and evaluation of pleural effusion.

Lung ultrasound has long been used for identification of pleural effusions. In our study the sensitivity $93.8 \%$ with accuracy $90 \%$.

There was a false positive result that has been shown in the lower lateral region.

\section{Consolidation:}

The sensitivity of lung ultrasound for the detection of consolidation in critically ill patients has been reported to be high, In our study lung ultrasound had a sensitivity of $93.3 \%$ and a diagnostic accuracy of $85 \%$ in identifying this abnormality. CXR had a much lower sensitivity $(53.3 \%)$ and diagnostic accuracy $(45 \%)$.

The lung ultrasound had two false positive cases, resulting in a specificity of $60 \%$, In all cases the consolidation was considered to be small in size and located in one region. It is likely that the time interval between lung ultrasound and $\mathrm{CT}$ (up to $4 \mathrm{~h}$ in some cases) as well as the mobilization and transportation of the patient to the radiology department contributes to this discrepancy (i.e., small consolidations may be resolved, particularly in mechanically ventilated patients). The nonuse of the shred sign, specific for consolidation, might also contribute to low specificity.

\section{Interstitial lung disease:}

In this study lung ultrasound had a sensitivity $(93.3 \%)$ and diagnostic accuracy of $90 \%$.

In one case lung ultrasound identified interstitial syndrome, where CT did not recognize such an abnormality (false positive case). The difference in time between the two imaging techniques might be the cause of this false positive case.

Thoracic CT was performed after lung ultrasound, within a time frame that for logistic reasons was between 1 and $4 \mathrm{~h}$. Studies have shown that this interval is sufficient to modify the appearance of interstitial syndrome on CT, because this pathology is very sensitive to treatment.

Lung ultrasound had also one false negative result, Nevertheless, lung ultrasound was much more sensitive and specific than bedside CXR in identifying interstitial syndrome.
In a study done by Xirouchaki et al. ${ }^{(7)}$, Lung ultrasound in critically ill patients in comparison with bedside chest radiography, To compare the diagnostic performance of lung ultrasound and bedside chest radiography (CXR) for the detection of various pathologic abnormalities in unselected critically ill patients, using thoracic computed tomography (CT) as a gold standard.

Forty-two mechanically ventilated patients scheduled for CT were prospectively studied with a modified lung ultrasound protocol. Four pathologic entities were evaluated: consolidation, interstitial syndrome, pneumothorax, and pleural effusion.

Each hemithorax was evaluated for the presence or absence of each abnormalityandthe patients were evaluated by the three imaging techniques.

\section{$\mathbf{1}^{\text {st }}$ consolidation}

The sensitivity, specificity, and diagnostic accuracy of CXR were 38, 89, and 49\%. The corresponding values for lung ultrasound were 100 , 78 , and $95 \%$, in our study values it was $93.3,60$, $80 \%$. So this study accepted that chest ultrasound is a better diagnostic tool with great sensitivity.

\section{$2^{\text {nd }}$ pneumothorax}

Ultrasound has a relatively low sensitivity $75 \%$, while our study showed a higher sensitivity $93.8 \%$. This difference may be due to The small number of cases and/or suboptimal methodology could explain the relatively low sensitivity.

\section{$3^{\text {rd }}$ pleural effusion:}

Sensitivity of chest ultrasound was $100 \%$, while in our study sensitivity was $93.8 \%$, so both study accepted that ultrasound is a better tool for diagnosis and evaluation of pleural effusion.

\section{$4^{\text {th }}$ interstitial lung disease}

Lung ultrasound had a sensitivity of $94 \%$, while our study is $93.3 \%$, so both study accepted that ultrasound is a better tool for diagnosis and evaluation of interstitial lung disease.

So the result that this study is closely agreed with our study that chest lung ultrasound has a considerably better diagnostic performance than CXR for the diagnosis of common pathologic conditions and may be used as an alternative to thoracic CT.

Also in another study done by Brogi et al. (8), Could the use of bedside lung ultrasound reduce the number of chest $\mathrm{x}$-rays in the intensive care unit?, aim was to evaluate the influence that introducing a routine daily use of lung ultrasound in critically ill patients may have on the number of CXRs and as a consequence, on medical costs and radiation exposure. 
This study divided patients into two groups:

A. In Group A, included patients admitted to the ICU from January 1, 2010 to May 31, 2012. During this period LUS was not yet implemented as a standard practice in our ICU and was only used sporadically on a consultancy basis. In this period, thoracic imaging was based on CXRs or thoracic $\mathrm{CT}$ scans as the standard of practice.

B. In Group B, included patients admitted to the ICU from June 1, 2012 to December 31, 2014. In this period, thoracic ultrasound was introduced in our ICU and implemented as the standard of care for many applications: physicians could choose to rely on LUS with or without integration with radiology methods. During that period, the imaging technique of choice was LUS, then, in case of clinical doubt or technical problems, X- Rays were used to overcome the issue.

This study shows the importance and effectiveness of LUS in reducing the number of CXRs performed in an academic polyvalent ICU. Routine LUS application, even when only left to the discretion of the caring physician allows decreasing the use of ionizing procedures as well as related biological and economic costs.

So also this study agreed with us that the use of chest ultrasound in critically ill patients can eliminate the risk of transportation of unstable patients and that of radiation exposure and is easily accessible in resource limited areas.

\section{Conclusion:}

Bedside chest ultrasound is a highly sensitive and specific tool in the detection and evaluation of many chest conditions that eliminates the risk of transportation of unstable patients and that of radiation exposure and is easily accessible in resource limited areas.

\section{References:}

1. Cardinale L, Priola AM, Moretti F, Volpicelli G (2014): Effectiveness of chest radiography, lung ultrasound and thoracic computed tomography in the diagnosis of congestive heart failure. World journal of radiology, 28;6(6):230.

2. Gargani $L$ and Picano E (2015): The risk of cumulative radiation exposure in chest imaging and the advantage of bedside ultrasound. Crit Ultrasound J., 2015;7:4.

3. Tapson VF (2012): Advances in the diagnosis and treatment of acute pulmonary embolism. Med Rep., 4:9. doi:10.3410/M4-9.

4. Mongodi S, Via G, Girard M, Rouquette I, Misset B, Braschi A, Mojoli F, Bouhemad B (2016): Chest Ultrasound for Early Diagnosis of Ventilator-Associated Pneumonia. Chest, 149(4):969-80.

5. Biasucci DG, Cutuli SL, Pizza A, Cavaliere F (2015): Clinically integrated ultrasound for decreasing the number of chest $\mathrm{x}$-rays in the intensive care unit: It is high time to move forward a "global" use of ultrasound. J Crit Care, 30(5):1137-8.

6. Mourgeon E, Lichtenstein D, Goldstein I, Cluzel P, Grenier P, Rouby JJ (2004): Comparative diagnostic performances of auscultation, chest radiography, and lung ultrasonography in acute respiratory distress syndrome. Anesthesiology, 100:9-15.

7. Xirouchaki N, Magkanas E, Vaporidi K, Kondili E, Plataki M, Patrianakos A, Akoumianaki E, Georgopoulos D (2011): Lung ultrasound in critically ill patients: comparison with bedside chest radiography. Intensive care medicine, 37(9):1488.

8. Brogi E, Bignami E, Sidoti A, Shawar M, Gargani L, Vetrugno L, Volpicelli G, Forfori F (2017): Could the use of bedside lung ultrasound reduce the number of chest $\mathrm{x}$ rays in the intensive care unit? Cardiovascular Ultrasound, 15(1):23. 